\title{
Ehrhart polynomials, simplicial polytopes, magic squares and a conjecture of Stanley
}

\author{
Dedicated to Richard Stanley on the occasion of his sixtieth birthday \\ By Christos A. Athanasiadis at Heraklion
}

\begin{abstract}
It is proved that for a certain class of integer polytopes $P$ the polynomial $h(t)$ which appears as the numerator in the Ehrhart series of $P$, when written as a rational function of $t$, is equal to the $h$-polynomial of a simplicial polytope and hence that its coefficients satisfy the conditions of the $g$-theorem. This class includes the order polytopes of graded posets, previously studied by Reiner and Welker, and the Birkhoff polytope of doubly stochastic $n \times n$ matrices. In the latter case the unimodality of the coefficients of $h(t)$, which follows, was conjectured by Stanley in 1983.
\end{abstract}

\section{Introduction}

Let $P$ be an $m$-dimensional convex polytope in $\mathbb{R}^{q}$ having integer vertices. We will be concerned with the function $i(P, r)$ counting integer points in the $r$-fold dilate of $P$. It is a fundamental result due to Ehrhart [3], [4] that $i(P, r)$ is a polynomial in $r$ of degree $m$, called the Ehrhart polynomial. Thus one can write

$$
\sum_{r \geqq 0} i(P, r) t^{r}=\frac{h_{0}+h_{1} t+\cdots+h_{d} t^{d}}{(1-t)^{m+1}}
$$

for certain integers $h_{0}, h_{1}, \ldots, h_{d}$. It was proved by Stanley [15] that the integers $h_{i}$ are nonnegative. In this paper we describe simple conditions on $P$ which imply that the sequence $\left(h_{0}, h_{1}, \ldots, h_{d}\right)$ is equal to the $h$-vector of a $d$-dimensional simplicial polytope. Such vectors are characterized by McMullen's $g$-theorem; see [1], [9], [14] and Section 2. In particular, they are symmetric and satisfy the inequalities

$$
h_{0} \leqq h_{1} \leqq \cdots \leqq h_{\lfloor d / 2\rfloor}
$$

Our main result (Theorem 3.5) applies to the Birkhoff polytope of doubly stochastic $n \times n$ matrices and to order polytopes of graded posets. In the former case $i(P, r)$ is equal to the 
number of $n \times n$ integer stochastic matrices, or magic squares, having line sums equal to $r$ and the inequalities (2) prove a long-standing conjecture of Stanley [16], Section I.1. In the latter case the integers $h_{i}$ count linear extensions of a naturally labeled poset by the number of descents and our result specializes to a recent result of Reiner and Welker [12]. Our method extends that in the work [12], which provided one of the main motivations for this paper. New elements in our approach are the concept of a 'special simplex', introduced in Section 3, and the employment of reverse lexicographic triangulations, which are combined to produce simplicial decompositions of integer polytopes more general than order polytopes of graded posets and to construct from these decompositions, as in [12], explicitly simplicial polytopes with prescribed $h$-vectors. In Section 4 we state several corollaries of our main result in the context of affine semigroup rings.

I am grateful to Volkmar Welker for encouraging e-discussions and to Jesús DeLoera, Victor Reiner, Francisco Santos, Richard Stanley and Bernd Sturmfels for helpful comments.

\section{Background}

In this section we review some basic definitions and background on convex polytopes and their face numbers, triangulations and Ehrhart polynomials. We refer the reader to the texts by Stanley [16], [17], Sturmfels [20] and Ziegler [21] for more information on these topics. We denote by $\mathbb{N}$ the set of nonnegative integers.

Face enumeration. Given a finite (abstract or geometric) simplicial complex $\Delta$ of dimension $d-1$, let $f_{i}$ denote the number of $i$-dimensional faces of $\Delta$, so that $\left(f_{0}, f_{1}, \ldots, f_{d-1}\right)$ is the $f$-vector of $\Delta$. The polynomial

$$
\sum_{i=0}^{d} f_{i-1}(x-1)^{d-i}=\sum_{i=0}^{d} h_{i} x^{d-i}
$$

where $f_{-1}=1$ unless $\Delta$ is empty, is the h-polynomial of $\Delta$, denoted $h(\Delta, t)$. The h-vector of $\Delta$ is the sequence $\left(h_{0}, h_{1}, \ldots, h_{d}\right)$ defined by (3).

A polytopal complex $\mathscr{F}$ [21], Section 8.1, is a finite, nonempty collection of convex polytopes such that (i) any face of a polytope in $\mathscr{F}$ is also in $\mathscr{F}$ and (ii) the intersection of any two polytopes in $\mathscr{F}$ is either empty or a face of both. The elements of $\mathscr{F}$ are its faces and those of dimension 0 are its vertices. The dimension of $\mathscr{F}$ is the maximum dimension of a face. The complex $\mathscr{F}$ is pure if all maximal faces of $\mathscr{F}$ have the same dimension. The collection $\mathscr{F}(P)$ of all faces of a polytope $P$ and the collection $\mathscr{F}(\partial P)$ of its proper faces are pure polytopal complexes called the face complex and boundary complex of $P$, respectively. Thus $P$ is simplicial if $\mathscr{F}(\partial P)$ is a simplicial complex. The $h$-vectors of boundary complexes of simplicial polytopes are characterized by McMullen's g-theorem [9], [16], Section III.1, [21], Section 8.6, as follows. A sequence $\left(g_{0}, g_{1}, \ldots, g_{\ell}\right)$ of nonnegative integers is said to be an $M$-vector if

(i) $g_{0}=1$ and

(ii) $0 \leqq g_{i+1} \leqq g_{i}^{\langle i\rangle}$ for $1 \leqq i \leqq \ell-1$, 
where $0^{\langle i\rangle}=0$ and

$$
n^{\langle i\rangle}=\left(\begin{array}{c}
k_{i}+1 \\
i+1
\end{array}\right)+\left(\begin{array}{c}
k_{i-1}+1 \\
i
\end{array}\right)+\cdots+\left(\begin{array}{c}
k_{j}+1 \\
j+1
\end{array}\right)
$$

for the unique representation

$$
n=\left(\begin{array}{c}
k_{i} \\
i
\end{array}\right)+\left(\begin{array}{c}
k_{i-1} \\
i-1
\end{array}\right)+\cdots+\left(\begin{array}{c}
k_{j} \\
j
\end{array}\right)
$$

with $k_{i}>k_{i-1}>\cdots>k_{j} \geqq j \geqq 1$, if $n \geqq 1$. A sequence $\left(h_{0}, h_{1}, \ldots, h_{d}\right)$ of nonnegative integers is the $h$-vector of the boundary complex of a $d$-dimensional simplicial polytope if and only if

(i) $h_{i}=h_{d-i}$ for all $i$ and

(ii) $\left(h_{0}, h_{1}-h_{0}, \ldots, h_{\lfloor d / 2\rfloor}-h_{\lfloor d / 2\rfloor-1}\right)$ is an $M$-vector.

In particular $\left(h_{0}, h_{1}, \ldots, h_{d}\right)$ is symmetric and unimodal and hence satisfies the inequalities (2), known as the Generalized Lower Bound Theorem for simplicial polytopes.

Triangulations and Ehrhart polynomials. A triangulation of a polytopal complex $\mathscr{F}$ is a geometric simplicial complex $\Delta$ with vertices those of $\mathscr{F}$ and underlying space equal to the union of the faces of $\mathscr{F}$, such that every maximal face of $\Delta$ is contained in a face of $\mathscr{F}$. A triangulation of the face complex $\mathscr{F}(P)$ of a polytope $P$ is simply called a triangulation of $P$.

For any set $\sigma$ consisting of vertices of the polytopal complex $\mathscr{F}$ we denote by $\mathscr{F} \backslash \sigma$ the subcomplex of faces of $\mathscr{F}$ which do not contain any of the vertices in $\sigma$ and write $\mathscr{F} \backslash v$ for $\mathscr{F} \backslash \sigma$ if $\sigma$ consists of a single vertex $v$. Given a linear ordering $\tau=\left(v_{1}, v_{2}, \ldots, v_{p}\right)$ of the set of vertices of $\mathscr{F}$ we define the reverse lexicographic triangulation or pulling triangulation $\Delta(\mathscr{F})=\Delta_{\tau}(\mathscr{F})$ with respect to $\tau[15],[8],[20]$, p. 67, as $\Delta(\mathscr{F})=\{v\}$ if $\mathscr{F}$ consists of a single vertex $v$ and

$$
\Delta(\mathscr{F})=\Delta\left(\mathscr{F} \backslash v_{p}\right) \cup \bigcup_{F}\left\{\operatorname{conv}\left(\left\{v_{p}\right\} \cup G\right): G \in \Delta(\mathscr{F}(F)) \cup\{\emptyset\}\right\}
$$

otherwise, where the union runs through the facets $F$ not containing $v_{p}$ of the maximal faces of $\mathscr{F}$ which contain $v_{p}$ and $\Delta\left(\mathscr{F} \backslash v_{p}\right)$ and $\Delta(\mathscr{F}(F))$ are defined with respect to the linear orderings of the vertices of $\mathscr{F} \backslash v_{p}$ and $F$, respectively, induced by $\tau$. Equivalently, for $i_{0}<i_{1}<\cdots<i_{t}$ the set $\left\{v_{i_{0}}, v_{i_{1}}, \ldots, v_{i_{t}}\right\}$ is the vertex set of a maximal simplex of $\Delta_{\tau}(\mathscr{F})$ if there exists a maximal flag $F_{0} \subset F_{1} \subset \cdots \subset F_{t}$ of faces of $\mathscr{F}$ such that $v_{i_{j}}$ is the last vertex of $F_{j}$ with respect to $\tau$ for all $j$ and $v_{i j}$ is not a vertex of $F_{j-1}$ for $j \geqq 1$. A different way to define $\Delta_{\tau}(\mathscr{F})$ is the following. For any vertex $v$ of $\mathscr{F}$ let

$$
\operatorname{pull}_{v}(\mathscr{F})=(\mathscr{F} \backslash v) \cup \bigcup_{F}\{\operatorname{conv}(\{v\} \cup G): G \in \mathscr{F}(F) \cup\{\emptyset\}\}
$$

where the union runs through the facets $F$ not containing $v$ of the maximal faces of $\mathscr{F}$ which contain $v$. If $\mathscr{F}_{0}=\mathscr{F}_{\text {and }} \mathscr{F}_{i}=\operatorname{pull}_{v_{p-i+1}}\left(\mathscr{F}_{i-1}\right)$ for $1 \leqq i \leqq p$ then $\widetilde{F}_{p}$ is a triangula- 
tion of $\mathscr{F}$ which coincides with $\Delta_{\tau}(\mathscr{F})$. It follows from [10], Theorem 2.5.23 (see also [6], p. 80) that if $\mathscr{F}$ is the boundary complex of a polytope $P$ then $\operatorname{pull}_{v}(\mathscr{F})$ is the boundary complex of another polytope, obtained from $P$ by moving its vertex $v$ beyond the hyperplanes supporting exactly those facets of $P$ which contain $v$. This observation implies the following lemma.

Lemma 2.1. The reverse lexicographic triangulation of the boundary complex of a polytope with respect to any ordering of its vertices is abstractly isomorphic to the boundary complex of a simplicial polytope of the same dimension.

A convex polytope $P \subseteq \mathbb{R}^{q}$ is said to be a rational or an integer polytope if all its vertices have rational or integer coordinates, respectively. It is called a $0 / 1$ polytope if all its vertices are $0 / 1$ vectors in $\mathbb{R}^{q}$. If $P$ is rational then the function defined for nonnegative integers $r$ by the formula

$$
i(P, r)=\#\left(r P \cap \mathbb{Z}^{q}\right)
$$

is a quasi-polynomial in $r$, called the Ehrhart quasi-polynomial of $P$ [17], Section 4.6. If $P$ is an integer polytope then this quasi-polynomial is actually a polynomial in $r$. Let $A \subseteq \mathbb{R}^{q}$ be the affine span of the integer polytope $P$. A triangulation $\Delta$ of $P$ is called unimodular if the vertex set of any maximal simplex of $\Delta$ is a basis of the affine integer lattice $A \cap \mathbb{Z}^{q}$. We denote by $\Delta_{\tau}$ the reverse lexicographic triangulation of an arbitrary polytope $P$ with respect to the ordering $\tau$ of its vertices. Following [15] we call such an ordering of the vertices of an integer polytope $P$ compressed if $\Delta_{\tau}$ is unimodular and call $P$ itself compressed if so is any linear ordering of its vertices. The following lemma holds for any unimodular triangulation of $P$, although we will not need this fact here.

Lemma 2.2 ([15], Corollary 2.5). If $P$ is an m-dimensional integer polytope in $\mathbb{R}^{q}$ and $\tau$ is a compressed ordering of its vertices then

$$
\sum_{r \geqq 0} i(P, r) t^{r}=\frac{h\left(\Delta_{\tau}, t\right)}{(1-t)^{m+1}}
$$

Two compressed polytopes. (a) A real $n \times n$ matrix is said to be doubly stochastic if all its entries are nonnegative and all its rows and columns sum to 1 . The set $P$ of all real doubly stochastic $n \times n$ matrices is a convex polytope in $\mathbb{R}^{n \times n}$ of dimension $(n-1)^{2}$, called the Birkhoff polytope [21], Example 0.12. It follows from the classical Birkhoff-von Neumann theorem that the vertices of $P$ are the $n \times n$ permutation matrices, so that $P$ is a $0 / 1$ polytope. The Birkhoff polytope was shown to be compressed by Stanley [15], Example 2.4 (b) (see also [20], Corollary 14.9).

(b) Let $\Omega$ be a poset (short for partially ordered set) on the ground set $[m]:=\{1,2, \ldots, m\}$. Recall that an (order) ideal of $\Omega$ is a subset $I \subseteq \Omega$ for which $i<_{\Omega} j$ and $j \in I$ imply that $i \in I$. Let $\Omega^{0}$ be the poset obtained from $\Omega$ by adjoining a minimum element 0 . The order polytope [18] of $\Omega$, denoted $O(\Omega)$, is the intersection of the hyperplane $x_{0}=1$ in $\mathbb{R}^{m+1}$ with the cone defined by the inequalities $x_{i} \geqq x_{j}$ for $i<j$ in $\Omega^{0}$ and $x_{i} \geqq 0$ for all $i$. Thus $O(\Omega)$ is an $m$-dimensional convex polytope. The vertices of $O(\Omega)$ are the characteristic vectors of the nonempty ideals of $\Omega^{0}$ [18], Corollary 1.3, so, in particular, $O(\Omega)$ is a $0 / 1$ polytope (see [18], Theorem 1.2, for a complete description of the facial struc- 
ture of $O(\Omega)$ ). Order polytopes were shown to be compressed by Ohsugi and Hibi [11], Example 1.3 (b).

Quotient polytopes. If $P \subseteq \mathbb{R}^{m}$ is an $m$-dimensional polytope and $V$ is any linear subspace of $\mathbb{R}^{m}$ then the quotient polytope $P / V \subseteq \mathbb{R}^{m} / V$ is the image of $P$ under the canonical surjection $\mathbb{R}^{m} \rightarrow \mathbb{R}^{m} / V$. This is a convex polytope in $\mathbb{R}^{m} / V$ linearly isomorphic to the image $\pi(P)$ of $P$ under any linear surjection $\pi: \mathbb{R}^{m} \rightarrow \mathbb{R}^{m-\operatorname{dim} V}$ with kernel $V$. Recall that the simplicial join $\Delta_{1} * \Delta_{2}$ of two abstract simplicial complexes $\Delta_{1}$ and $\Delta_{2}$ on disjoint vertex sets has faces the sets of the form $\sigma_{1} \cup \sigma_{2}$, where $\sigma_{1} \in \Delta_{1}$ and $\sigma_{2} \in \Delta_{2}$ and that $h\left(\Delta_{1} * \Delta_{2}, t\right)=h\left(\Delta_{1}, t\right) h\left(\Delta_{2}, t\right)$. The following proposition is essentially Proposition 3.12 in [12].

Proposition 2.3. Let $P$ be an m-dimensional polytope in $\mathbb{R}^{m}$ having a triangulation abstractly isomorphic to $\sigma * \Delta$, where $\sigma$ is the vertex set of a simplex not contained in the boundary of $P$. Let $V$ be the linear subspace of $\mathbb{R}^{m}$ parallel to the affine span of $\sigma$.

The boundary complex of the quotient polytope $P / V \subseteq \mathbb{R}^{m} / V$ is abstractly isomorphic to $\mathscr{F}(P) \backslash \sigma$ and inherits a triangulation abstractly isomorphic to $\Delta$.

\section{Special simplices}

Throughout this section $P$ denotes an $m$-dimensional convex polytope in $\mathbb{R}^{q}$ with face complex $\mathscr{F}(P)$. Let $\Sigma$ be a simplex spanned by $n$ vertices of $P$. We call $\Sigma$ a special simplex in $P$ if each facet of $P$ contains exactly $n-1$ of the vertices of $\Sigma$. Note that, in particular, $\Sigma$ is not contained in the boundary of $P$.

Example 3.1. Let $P$ be the polytope of real doubly stochastic $n \times n$ matrices. If $v_{1}, v_{2}, \ldots, v_{n}$ are the $n \times n$ permutation matrices corresponding to the elements of the cyclic subgroup of the symmetric group generated by the cycle $\left(\begin{array}{llll}1 & 2 & \cdots\end{array}\right)$ (or any $n$ permutation matrices with pairwise disjoint supports) then $v_{1}, v_{2}, \ldots, v_{n}$ are the vertices of a special simplex in $P$. Indeed, each facet of $P$ is defined by an equation of the form $x_{i j}=0$ in $\mathbb{R}^{n \times n}$ and misses exactly one of $v_{1}, v_{2}, \ldots, v_{n}$.

Example 3.2. Let $\Omega$ be a poset on the ground set $[m]:=\{1,2, \ldots, m\}$ which is graded of rank $n-2$ (we refer to [17], Chapter 3, for basic background and terminology on partially ordered sets) and $P=O(\Omega)$ be the order polytope of $\Omega$ in $\mathbb{R}^{m+1}$. Let $\Omega^{0}$ be the poset obtained from $\Omega$ by adjoining a minimum element 0 and for $1 \leqq i \leqq n$ let $v_{i}$ be the characteristic vector of the ideal of elements of $\Omega^{0}$ of rank at most $i-1$, so that $v_{i}$ is a vertex of $P$. Since a facet of $P$ is defined either by an equation of the form $x_{i}=x_{j}$ with $i<j$ in $\Omega^{0}$ and $i, j$ in successive ranks or by one of the form $x_{i}=0$ for $i \in \Omega^{0}$ of rank $n-1$, it follows that $v_{1}, v_{2}, \ldots, v_{n}$ are the vertices of a special simplex in $P$.

Lemma 3.3. Suppose that $v_{1}, v_{2}, \ldots, v_{n}$ are the vertices of a special simplex in $P$. If $F$ is a face of $P$ of codimension $k$ for some $1 \leqq k \leqq n-1$ and $F$ does not contain any of $v_{1}, v_{2}, \ldots, v_{k}$ then $F$ must contain $v_{i}$ for all $k+1 \leqq i \leqq n$.

Proof. Let $\Sigma$ be the special simplex with vertices $v_{1}, v_{2}, \ldots, v_{n}$. Any codimension $k$ face of a polytope can be written as the intersection of $k$ facets, so we can write 
$F=F_{1} \cap F_{2} \cap \cdots \cap F_{k}$ where the $F_{j}$ are facets of $P$. For each $1 \leqq i \leqq k$ we have $v_{i} \notin F$ and hence $v_{i} \notin F_{j}$ for some $j=j_{i}$. Since $\Sigma$ is special the integers $j_{1}, j_{2}, \ldots, j_{k}$ are all distinct and hence for each $1 \leqq j \leqq k$ we have $v_{i} \notin F_{j}$ for some $1 \leqq i \leqq k$, which in turn implies that $v_{i} \in F_{j}$ for all $k+1 \leqq i \leqq n$. It follows that $v_{i} \in F_{1} \cap F_{2} \cap \cdots \cap F_{k}=F$ for all $k+1 \leqq i \leqq n$.

Lemma 3.4. Suppose that $\tau=\left(v_{p}, v_{p-1}, \ldots, v_{1}\right)$ is an ordering of the vertices of $P$ such that $\sigma=\left\{v_{1}, v_{2}, \ldots, v_{n}\right\}$ is the vertex set of a special simplex in P. Let $\Delta$ be the abstract simplicial complex on $\left\{v_{n+1}, \ldots, v_{p}\right\}$ defined by the reverse lexicographic triangulation of $\mathscr{F}(P) \backslash \sigma$ with respect to $\left(v_{p}, v_{p-1}, \ldots, v_{n+1}\right)$.

(i) The reverse lexicographic triangulation $\Delta_{\tau}$ of $P$ is abstractly isomorphic to the simplicial join $\sigma * \Delta$.

(ii) $\Delta$ is abstractly isomorphic to the boundary complex of a simplicial polytope of dimension $m-n+1$.

Proof. (i) Let $\sigma_{i}=\left\{v_{1}, \ldots, v_{i}\right\}$ for $0 \leqq i \leqq n$, so that $\sigma_{0}=\emptyset$ and $\sigma_{n}=\sigma$, and let $\Delta_{i}$ denote the abstract simplicial complex on the set $\left\{v_{i+1}, \ldots, v_{p}\right\}$ defined by the reverse lexicographic triangulation of $\mathscr{F}(P) \backslash \sigma_{i}$ with respect to the ordering $\left(v_{p}, v_{p-1}, \ldots, v_{i+1}\right)$. To prove that $\Delta_{0}=\sigma_{n} * \Delta_{n}$, which is the assertion in the lemma, we will prove that $\mathscr{F}(P) \backslash \sigma_{i}$ is pure $(m-i)$-dimensional and that $\Delta_{0}=\sigma_{i} * \Delta_{i}$ for all $0 \leqq i \leqq n$ by induction on $i$. This is obvious for $i=0$ so let $1 \leqq i \leqq n$. By induction, any maximal face $F$ of $\mathscr{F}(P) \backslash \sigma_{i-1}$ is a codimension $i-1$ face of $P$. Since $F$ does not contain any of the vertices $v_{1}, \ldots, v_{i-1}$, by Lemma 3.3 we have $v_{i} \in F$. This implies that $\mathscr{F}(P) \backslash \sigma_{i}$ is pure $(m-i)$-dimensional and that $\Delta_{i-1}=v_{i} * \Delta_{i}$. The last equality and the induction hypothesis $\Delta_{0}=\sigma_{i-1} * \Delta_{i-1}$ imply that $\Delta_{0}=\sigma_{i} * \Delta_{i}$, which completes the induction.

(ii) Let $V$ be the linear subspace of $\mathbb{R}^{q}$ parallel to the affine span of the vertices in $\sigma$ and $P / V$ be the corresponding quotient polytope of $P$, so that $P / V$ has dimension $m-n+1$. Part (i) and Proposition 2.3 imply that $\Delta$ is abstractly isomorphic to a reverse lexicographic triangulation of the boundary complex of $P / V$. This is in turn isomorphic to the boundary complex of a simplicial polytope of dimension $m-n+1$ by Lemma 2.1 .

The following theorem is the main result in this paper.

Theorem 3.5. Suppose that $P$ is an integer polytope and $\tau=\left(v_{p}, v_{p-1}, \ldots, v_{1}\right)$ is an ordering of its vertices such that:

(i) $\tau$ is compressed and

(ii) $\left\{v_{1}, v_{2}, \ldots, v_{n}\right\}$ is the vertex set of a special simplex in $P$.

Then

$$
\sum_{r \geqq 0} i(P, r) t^{r}=\frac{h(t)}{(1-t)^{m+1}}
$$


where $h(t)=h_{0}+h_{1} t+\cdots+h_{d} t^{d}$ is the h-polynomial of the boundary complex of a simplicial polytope $Q$ of dimension $d=m-n+1$, so that $h(t)$ satisfies the conditions in the g-theorem.

In particular $h_{i}=h_{d-i}$ for all $i$ and $1=h_{0} \leqq h_{1} \leqq \cdots \leqq h_{\lfloor d / 2\rfloor}$.

Moreover, $Q$ can be chosen so that its boundary complex is abstractly isomorphic to the reverse lexicographic triangulation of $\mathscr{F}(P) \backslash\left\{v_{1}, \ldots, v_{n}\right\}$ with respect to the ordering $\left(v_{p}, v_{p-1}, \ldots, v_{n+1}\right)$.

Proof. Let $\sigma=\left\{v_{1}, v_{2}, \ldots, v_{n}\right\}$ and let $\Delta$ denote the reverse lexicographic triangulation of $\mathscr{F}(P) \backslash \sigma$ with respect to the ordering $\left(v_{p}, v_{p-1}, \ldots, v_{n+1}\right)$. Lemma 2.2 guarantees that the proposed equation holds with $h(t)=h\left(\Delta_{\tau}, t\right)$. Part (i) of Lemma 3.4 implies that

$$
h\left(\Delta_{\tau}, t\right)=h(\sigma * \Delta, t)=h(\sigma, t) h(\Delta, t)=h(\Delta, t)
$$

since face complexes of simplices have $h$-polynomial equal to 1 , and the result follows from part (ii) of the same lemma.

We now apply Theorem 3.5 to the Birkhoff polytope and to order polytopes of graded posets. Observe that our theorem does not apply to all integer polytopes, for instance to nonunimodular integer simplices.

Magic squares and the Birkhoff polytope. An integer stochastic matrix, or magic square, is a square matrix with nonnegative integer entries having all line sums equal to each other, where a line is a row or a column. Let $H_{n}(r)$ be the number of $n \times n$ magic squares with line sums equal to $r$. The fact that for any fixed positive integer $n$ the quantity $H_{n}(r)$ is a polynomial in $r$ of degree $(n-1)^{2}$ was first proved by Stanley [13] (see also [5], [16], Section I.5, and [17], Section 4.6). Let $P$ be the polytope of real doubly stochastic $n \times n$ matrices and observe that $H_{n}(r)$ coincides with the Ehrhart polynomial $i(P, r)$. Since $P$ is a compressed integer polytope of dimension $(n-1)^{2}$, Theorem 3.5 and Example 3.1 imply immediately the following corollary.

Corollary 3.6. For any positive integer $n$ we have

$$
\sum_{r \geqq 0} H_{n}(r) t^{r}=\frac{h(t)}{(1-t)^{(n-1)^{2}+1}}
$$

where $h(t)=h_{0}+h_{1} t+\cdots+h_{d} t^{d}$ is the h-polynomial of the boundary complex of a simplicial polytope of dimension $d=n^{2}-3 n+2$, so that $h(t)$ satisfies the conditions in the g-theorem.

$$
\text { In particular } h_{i}=h_{d-i} \text { for all } i \text { and } 1=h_{0} \leqq h_{1} \leqq \cdots \leqq h_{\lfloor d / 2\rfloor} \text {. }
$$

In view of the last statement in Theorem 3.5, the polytope in the previous corollary can be constructed by pulling in an arbitrary order the vertices of the quotient of $P$ with respect to the affine span of the vertices $v_{1}, v_{2}, \ldots, v_{n}$, chosen explicitly as in Example 3.1. 
Eulerian polynomials and equatorial spheres. Let $\Omega$ be a graded poset on the ground set $[m]:=\{1,2, \ldots, m\}$ of rank $n-2$. Let $\Omega_{i}$ be the set of elements of $\Omega$ of rank $i-1$ for $1 \leqq i \leqq n-1$ and $\mathscr{L}(\Omega)$ be the set of linear extensions of $\Omega$, meaning the set of permutations $w=\left(w_{1}, w_{2}, \ldots, w_{m}\right)$ of $[m]$ for which $w_{i}<_{\Omega} w_{j}$ implies $i<j$. We assume that $\Omega$ is naturally labeled, meaning that the identity permutation $(1,2, \ldots, m)$ is a linear extension. The $\Omega$-Eulerian polynomial is defined as

$$
W(\Omega, t)=\sum_{w \in \mathscr{L}(\Omega)} t^{\operatorname{des}(w)}
$$

where

$$
\operatorname{des}(w)=\#\left\{i \in[m-1]: w_{i}>w_{i+1}\right\}
$$

is the number of descents of $w$. Following [12] we call a function $g: \Omega \rightarrow \mathbb{R}$ equatorial if $\min _{a \in \Omega} g(a)=0$ and for each $2 \leqq i \leqq n-1$ there exist $a_{i-1} \in \Omega_{i-1}$ and $a_{i} \in \Omega_{i}$ such that $a_{i-1}<_{\Omega} a_{i}$ and $g\left(a_{i-1}\right)=g\left(a_{i}\right)$. An ideal $I$ or, more generally, a strictly increasing chain of ideals $I_{1} \subset I_{2} \subset \cdots \subset I_{k}$ in $\Omega$ is equatorial if the characteristic function $\chi_{I}$ of $I$ or the sum $\chi_{I_{1}}+\chi_{I_{2}}+\cdots+\chi_{I_{k}}$, respectively, is equatorial. The equatorial complex $\Delta_{\text {eq }}(\Omega)$, introduced in [12], is the abstract simplicial complex on the vertex set of equatorial ideals of $\Omega$ whose simplices are the equatorial chains of ideals in $\Omega$.

The following theorem is proved in Corollary 3.8 and Theorem 3.14 of [12].

Theorem 3.7 (Reiner-Welker [12]). Let $\Omega$ be a naturally labeled, graded poset on $[\mathrm{m}]$ having $n-1$ ranks. The equatorial complex $\Delta_{\mathrm{eq}}(\Omega)$ is abstractly isomorphic to the boundary complex of a simplicial polytope of dimension $d=m-n+1$ which has h-polynomial equal to the $\Omega$-Eulerian polynomial $W(\Omega, t)$.

Hence $W(\Omega, t)$ satisfies the conditions in the g-theorem and, in particular, it has symmetric and unimodal coefficients.

Let $P$ be the order polytope of $\Omega$ and $\Omega^{0}$ be the poset obtained from $\Omega$ by adjoining a minimum element 0 . Recall that the vertices of $P$ are the characteristic vectors of the nonempty ideals of $\Omega^{0}$. The order polytope comes with its canonical triangulation [18], [12], Proposition 2.1, which is a unimodular triangulation with maximal simplices bijecting to the linear extensions of $\Omega$. This canonical triangulation is in fact the reverse lexicographic triangulation of $O(\Omega)$ with respect to any ordering $\left(u_{p}, u_{p-1}, \ldots, u_{1}\right)$ of its vertices such that $i<j$ whenever the ideal of $\Omega^{0}$ defined by $u_{i}$ is strictly contained in that defined by $u_{j}$. We will use the following lemma.

Lemma 3.8. Let $v_{i}$ be the characteristic vector of the ideal of elements of $\Omega^{0}$ of rank at most $i-1$ for $1 \leqq i \leqq n$. Let $\sigma=\left\{v_{1}, \ldots, v_{n}\right\}$ and $\tau=\left(v_{p}, \ldots, v_{n+1}\right)$ be an ordering of the remaining vertices of $P$ such that $i<j$ whenever $i, j \geqq n+1$ and the ideal defined by $v_{i}$ is strictly contained in that defined by $v_{j}$.

The equatorial complex $\Delta_{\mathrm{eq}}(\Omega)$ is the abstract simplicial complex defined by the reverse lexicographic triangulation of $\mathscr{F}(P) \backslash \sigma$ with respect to $\tau$. 
Proof. Let $\mathscr{F}$ denote the face complex of $P$ and let $x_{\hat{1}}=0$ by convention. The maximal faces of $\mathscr{F} \backslash \sigma$ are the faces of $P$ defined by systems of equations of the form $x_{i_{s}}=x_{j_{s}}$ for $0 \leqq s \leqq n-1$ where (i) $i_{0}=0$ and $j_{n-1}=\hat{1}$, (ii) $i_{s} \in \Omega_{s}$ for $1 \leqq s \leqq n-1, j_{s} \in \Omega_{s+1}$ for $0 \leqq s \leqq n-2$ and $i_{s}<_{\Omega} j_{s}$ for $1 \leqq s \leqq n-2$ and (iii) if $j_{s}=i_{s+1}$ for consecutive values $s=a, a+1, \ldots, b-1$ of $s$ then the interval $\left[i_{a}, j_{b}\right]$ in $\hat{\Omega}$ consists only of the elements of the chain $i_{a}<i_{a+1}<\cdots<i_{b}<j_{b}$. The statement of the lemma follows from the description of the maximal faces of a reverse lexicographic triangulation $\Delta(\mathscr{F})$ (see Section 2 ) and that of the maximal faces of $\Delta_{\text {eq }}(\Omega)$ (see [12], Proposition 3.5). We omit the details.

Proof of Theorem 3.7. Let $P$ be the order polytope of $\Omega$, as before. Observe that $i(P, r)$ is equal to the number of order reversing maps $\rho: \Omega \rightarrow\{0,1, \ldots, r\}$. It follows from [17], Theorem 4.5.14, that

$$
\sum_{r \geqq 0} i(P, r) t^{r}=\frac{W(\Omega, t)}{(1-t)^{m+1}}
$$

Let the vertices $v_{1}, v_{2}, \ldots, v_{p}$ of $P$ and $\tau=\left(v_{p}, \ldots, v_{n+1}\right)$ be as in Lemma 3.8. We checked in Example 3.2 that $v_{1}, v_{2}, \ldots, v_{n}$ are the vertices of a special simplex in $P$. Since $P$ is a compressed integer polytope (see Section 2) Theorem 3.5 applies and we have

$$
\sum_{r \geqq 0} i(P, r) t^{r}=\frac{h(t)}{(1-t)^{m+1}}
$$

where $h(t)=h_{0}+h_{1} t+\cdots+h_{d} t^{d}$ is the $h$-polynomial of a simplicial polytope of dimension $d=m-n+1$ having, in view of Lemma 3.8, boundary complex abstractly isomorphic to $\Delta_{\text {eq }}(\Omega)$. Comparison with (4) yields $h(t)=W(\Omega, t)$ and completes the proof.

\section{Rational polyhedral cones and semigroup rings}

In this section we consider the affine semigroup ring corresponding to an integer polytope and state several corollaries of Theorem 3.5, including a generalization of Corollary 3.6 to magic labelings of bipartite graphs. For terminology and background related to semigroup rings we refer the reader to [2], Chapter 6.

Let $P$ be an $m$-dimensional integer polytope in $\mathbb{R}^{q}$. We denote by $R_{P}$ the subalgebra of the algebra $\mathbf{k}\left[x_{1}, \ldots, x_{q}, x_{1}^{-1}, \ldots, x_{q}^{-1}, t\right]$ of Laurant polynomials over a field $\mathbf{k}$ generated by the monomials $x^{\alpha} t^{r}$ for positive integers $r$ and $\alpha \in \mathbb{Z}^{q}$ such that $\alpha / r \in P$. The algebra $R_{P}$ can be graded by letting $x^{\alpha} t^{r}$ have degree $r$. With this grading $R_{P}$ is a normal, CohenMacaulay, graded commutative ring whose Hilbert series is the Ehrhart series of $P$. Let $\tilde{P}=\{(1, x): x \in P\}$ be the lift of $P$ in the hyperplane $x_{0}=1$ in $\mathbb{R}^{q+1}$. We denote by $\mathscr{C}_{P}$ the cone in $\mathbb{R}^{q+1}$ generated by $\tilde{P}$ and by $E_{P}$ the semigroup of integer points in $\mathscr{C}_{P}$. We also denote by $\bar{E}_{P}$ the set of points of $E_{P}$ which lie in the relative interior of $\mathscr{C}_{P}$. It is known that the ring $R_{P}$ is Gorenstein if and only if $\bar{E}_{P}$ has a unique minimal element, meaning an element $\beta \in \bar{E}_{P}$ such that $\bar{E}_{P}=\beta+E_{P}$; see for instance [2], Corollary 6.3.8.

Corollary 4.1. Let $P$ be an $m$-dimensional integer polytope in $\mathbb{R}^{q}$. If

(i) $R_{P}$ is Gorenstein and 
(ii) there exists a compressed ordering $\tau=\left(v_{p}, v_{p-1}, \ldots, v_{1}\right)$ of the vertices of $\tilde{\boldsymbol{P}}$ such that $v_{1}+v_{2}+\cdots+v_{n}$ is equal to the unique minimal element $\beta$ of $\bar{E}_{P}$ for some $n$,

then the conclusion of Theorem 3.5 holds.

Proof. We denote by $\mathscr{L}$ the linear span of $\mathscr{C}_{P}$ in $\mathbb{R}^{q+1}$ and by $\mathscr{A}$ a set of integer linear forms in $\mathbb{R}^{q+1}$, one for each facet of $P$, defining the cone $\mathscr{C}_{P}$ as the set of points $x \in \mathscr{L}$ satisfying $g(x) \geqq 0$ for all $g \in \mathscr{A}$. In view of Theorem 3.5 it suffices to show that $\left\{v_{1}, v_{2}, \ldots, v_{n}\right\}$ is the vertex set of a special simplex in $\tilde{P}$. Let $F$ be a facet of $\tilde{P}$ and let $f$ be the linear form in $\mathscr{A}$ corresponding to $F$. We need to show that exactly one of $v_{1}, v_{2}, \ldots, v_{n}$ satisfies $f\left(v_{i}\right)>0$. Clearly at least one of them has this property, since $f(\beta)>0$. Assume that at least two of $v_{1}, v_{2}, \ldots, v_{n}$ satisfy $f\left(v_{i}\right)>0$, say $v_{j}$ is one of them, and let $f(\beta)=b$ and $f\left(v_{j}\right)=c$, so that $1 \leqq c<b$. Since $F$ is a facet of $\tilde{P}$ there exists a point $x$ in the affine span of $\tilde{P}$, which we may assume to have rational coordinates, satisfying $f(x)<0$ and $g(x)>0$ for all $g \in \mathscr{A}$ other than $f$. By replacing $x$ with a suitable positive integer multiple we find an integer point $\alpha$ in $\mathscr{L}$ satisfying $f(\alpha)<0$ and $g(\alpha)>0$ for all $g \in \mathscr{A}$ other than $f$. Letting $a=f(\alpha)$, we may choose a nonnegative integer $t$ so that $0<a+b+t c<b$. Then $\gamma=\alpha+\beta+t v_{j}$ is in $\bar{E}_{P}$ and satisfies $f(\gamma)<f(\beta)$, which contradicts the minimality of $\beta$.

Recall that $R_{P}$ is standard if it is generated by its homogeneous elements of degree one or, equivalently, if $E_{P}$ is generated as a semigroup by the integer points in $\tilde{P}$. Clearly this holds if $P$ has a unimodular triangulation. The next corollary provides an instance in which a conjecture of Hibi [7], Conjecture 1.5, and Stanley (see [19], Conjecture 4a), stating that the $h$-vector of a standard, graded, Gorenstein domain has unimodal coefficients, can be answered in the affirmative.

Corollary 4.2. Let $P$ be an m-dimensional integer polytope in $\mathbb{R}^{q}$. If

(i) $P$ is compressed and

(ii) $R_{P}$ is Gorenstein,

then the conclusion of Theorem 3.5 holds where, in the statement of the theorem, $n$ is the $x_{0}$-coordinate of the unique minimal element of $\bar{E}_{P}$.

Proof. Let $\beta$ be the unique minimal element of $\bar{E}_{P}$, whose existence is guaranteed by (ii). Since $\tilde{P}$ has unimodular triangulations (having vertex set that of $\tilde{P}$ ), the vertices of $\tilde{P}$ must be its only integer points. Hence the fact that $R_{P}$ is standard implies that $\beta=v_{1}+v_{2}+\cdots+v_{n}$ for some vertices $v_{1}, v_{2}, \ldots, v_{n}$ of $\tilde{P}$, which must be pairwise distinct by the minimality of $\beta$. Because of (i) any ordering $\left(v_{p}, v_{p-1}, \ldots, v_{1}\right)$ of the vertices of $\tilde{P}$ satisfies the assumptions of Corollary 4.1. The result follows from this corollary observing that $\beta$ has $x_{0}$-coordinate equal to $n$.

General conditions on $P$ which guarantee that $P$ is compressed were given by Ohsugi and Hibi [11]. Let $\Phi$ be a $p \times q$ matrix with integer entries. Let $u \in \mathbb{Z}^{p}$ and suppose that $P$ is defined by the linear equalities and inequalities

$$
\Phi x=u \text { and } 0 \leqq x_{i} \leqq 1 \quad \text { for } 1 \leqq i \leqq q,
$$

where $x=\left(x_{1}, x_{2}, \ldots, x_{q}\right) \in \mathbb{R}^{q}$. 
Corollary 4.3. Let $P$ be an $m$-dimensional polytope, as in (5). If $P$ is a $0 / 1$ polytope and $R_{P}$ is Gorenstein then the conclusion of Corollary 4.2 holds.

Proof. Since $P$ is a $0 / 1$ polytope, Theorem 1.1 in [11] implies that $P$ is compressed. Thus the assumptions of Corollary 4.2 hold.

Magic labelings of graphs. Let $G$ be a graph (multiple edges and loops allowed) with $p$ vertices and $q$ edges and edge set $\mathscr{E}$. A magic labeling [13] of $G$ of index $r$ is an assignment $\ell: \mathscr{E} \rightarrow \mathbb{N}$ of nonnegative integers to the edges of $G$ such that for each vertex $v$ of $G$ the sum of the labels of all edges incident to $v$ is equal to $r$, in other words,

$$
\sum_{e: v \in e} \ell(e)=r
$$

Let $\mathbb{R}^{\mathscr{E}}$ denote the real vector space with basis $\mathscr{E}$ and let $x_{e}$ be the linear functional on $\mathbb{R}^{\mathscr{E}}$ dual to the basis element $e \in \mathscr{E}$. Let $P$ be the set of points $x \in \mathbb{R}^{\mathscr{E}}$ satisfying $x_{e} \geqq 0$ for all $e \in \mathscr{E}$ and

$$
\sum_{e: v \in e} x_{e}=1
$$

for all vertices $v$ of $G$. Thus $P$ is a convex polytope in $\mathbb{R}^{\mathscr{E}}$ and $i(P, r)$ counts the number $H_{G}(r)$ of magic labelings of $G$ of index $r$. It follows from [13], Proposition 2.9, and either [15], Theorem 2.3 (applied as in [15], Example 2.4 (b), in the case of the Birkhoff polytope) or [11], Theorem 1.1, that $P$ is a compressed integer polytope, if the graph $G$ is bipartite (or, more generally, if it satisfies condition (iii) of [13], Proposition 2.9). Clearly $R_{P}$ is Gorenstein if $G$ is regular of degree $n$, since then $(n, 1,1, \ldots, 1)$ is the unique minimal element of $\bar{E}_{P}$. Assuming further that $G$ is connected, $P$ has dimension $m=q-p+1$. The following corollary specializes to Corollary 3.6 when $G$ is the complete bipartite graph on two sets of vertices, each of size $n$.

Corollary 4.4. For $n \geqq 1$ and for any connected regular bipartite graph $G$ with $p$ vertices and $q=n p / 2$ edges we have

$$
\sum_{r \geqq 0} H_{G}(r) t^{r}=\frac{h(t)}{(1-t)^{m+1}}
$$

where $m=q-p+1$ and $h(t)=h_{0}+h_{1} t+\cdots+h_{d} t^{d}$ is the h-polynomial of the boundary complex of a simplicial polytope of dimension $d=m-n+1$, so that $h(t)$ satisfies the conditions in the g-theorem.

In particular $h_{i}=h_{d-i}$ for all $i$ and $1=h_{0} \leqq h_{1} \leqq \cdots \leqq h_{\lfloor d / 2\rfloor}$.

\section{References}

[1] L. J. Billera and C.W. Lee, A proof of the sufficiency of the McMullen conditions for $f$-vectors of simplicial polytopes, J. Combin. Th. (A) 31 (1981), 237-255.

[2] W. Bruns and J. Herzog, Cohen-Macaulay rings, Cambridge Stud. Adv. Math. 39, Cambridge University Press, Cambridge 1998. 
[3] E. Ehrhart, Sur un problème de géométrie diophantienne linéaire. I. Polyèdres et réseaux, J. reine angew. Math. 226 (1967), 1-29.

[4] E. Ehrhart, Sur un problème de géométrie diophantienne linéaire. II. Systèms diophantiens linéaires, J. reine angew. Math. 227 (1967), 25-49.

[5] E. Ehrhart, Sur les carrés magiques, C. R. Acad. Sci. Paris 227 A (1973), 575-577.

[6] B. Grünbaum, Convex Polytopes, Wiley-Interscience, New York 1967; second edition, Grad. Texts Math. 221, Springer-Verlag, New York 2002.

[7] T. Hibi, Flawless $O$-sequences and Hilbert functions of Cohen-Macaulay integral domains, J. Pure Appl. Algebra 60 (1989), 245-251.

[8] C. W. Lee, Regular triangulations of convex polytopes, in: Applied Geometry and Discrete MathematicsThe Victor Klee Festschrift, P. Gritzmann and B. Sturmfels, eds., Amer. Math. Soc., DIMACS Series 4, Providence, RI (1991), 443-456.

[9] P. McMullen, The number of faces of simplicial polytopes, Israel J. Math. 9 (1971), 559-570.

[10] P. McMullen and G. C. Shephard, Convex Polytopes and the Upper Bound Conjecture, London Math. Soc. Lect. Note Ser. 3, Cambridge University Press, London/New York 1971.

[11] H. Ohsugi and T. Hibi, Convex polytopes all of whose reverse lexicographic initial ideals are squarefree, Proc. Amer. Math. Soc. 129 (2001), 2541-2546.

[12] V. Reiner and V. Welker, On the Charney-Davis and Neggers-Stanley conjectures, J. Combin. Th. (A) 109 (2005), 247-280.

[13] R. P. Stanley, Linear homogeneous Diophantine equations and magic labelings of graphs, Duke Math. J. 40 (1973), 607-632.

[14] R. P. Stanley, The number of faces of a simplicial convex polytope, Adv. Math. 35 (1980), 236-238.

[15] R. P. Stanley, Decompositions of rational convex polytopes, Ann. Discr. Math. 6 (1980), 333-342.

[16] R. P. Stanley, Combinatorics and Commutative Algebra, Progr. Math. 41, Birkhäuser, Boston, first edition 1983; second edition 1996.

[17] R. P. Stanley, Enumerative Combinatorics, vol. 1, Wadsworth \& Brooks/Cole, Pacific Grove, CA 1986; second printing, Cambridge University Press, Cambridge 1997.

[18] R. P. Stanley, Two poset polytopes, Discr. Comput. Geom. 1 (1986), 9-23.

[19] R. P. Stanley, Log-concave and unimodal sequences in algebra, combinatorics, and geometry, in: Graph Theory and its Applications: East and West, Ann. New York Acad. Sci. 576, New York Acad. Sci., New York (1989), 500-535.

[20] B. Sturmfels, Gröbner Bases and Convex Polytopes, Univ. Lect. Ser. 8, American Mathematical Society, Providence, RI, 1996.

[21] G. M. Ziegler, Lectures on Polytopes, Grad. Texts Math. 152, Springer-Verlag, New York 1995.

Department of Mathematics, University of Crete, 71409 Heraklion, Crete, Greece e-mail: caa@math.uoc.gr

Eingegangen 8. März 2004 